KCL-PH-TH/2013-01

LCTS/2012-34

\title{
On Gravitino properties in a Conformal Supergravity Model
}

\author{
Nick E. Mavromatos \\ Theoretical Particle Physics and Cosmology Group, Department of Physics, \\ King's College London, Strand, London WC2R 2LS, UK; \\ Theory Division, Physics Department, CERN, CH-1211 Geneva 23, Switzerland \\ Vassilis C. Spanos \\ Institute of Nuclear Physics, NCSR "Demokritos", GR-15310 Athens, Greece
}

\begin{abstract}
In the context of a conformal Supergravity (SUGRA) model in the Einstein frame, in which the (next to) minimal supersymmetric standard model can embedded naturally to produce chaotic inflation scenarios, we study properties of gravitino in the cases where it is stable or unstable. In the latter case, we demonstrate that for large dilaton scale factors there is an enhanced magnitude of the gravitino width, when it decays to neutralino dark matter, as compared with the standard SUGRA case. In this context, we discuss the associated consequences as far as Big Bang Nucleosynthesis constraints and avoidance of gravitino overproduction are concerned.
\end{abstract}

\section{INTRODUCTION}

In [1, 2] simple classes of supergravity (SUGRA) models describing superconformal coupling of matter to supergravity have been considered. The models contain non-minimal scalar/space-time-curvature couplings of the form $\Phi R$, where $\Phi$ is a frame function, depending in general on matter supermultiplets, including dilatons. Such couplings have been argued to lead naturally to Higgs-inflation in both non-supersymmetric [36] and supersymmetric theories [7 9]. Scale-free globally supersymmetric theories, such as the Next to Minimal Supersymmetric Standard Model (NMSSM) [10] with a scale-invariant superpotential, can be naturally embedded [2, 7] into this class of theories, leading to new classes of chaotic inflationary scenarios 1]. Moreover, such models have been considered in 11] in connection with the possibility of dynamical breaking of supergravity theories, exploring further the conformal couplings of the gravitino four-fermion interactions.

It is the point of this article to discuss the properties of gravitino fields in such models, in particular in the context of the NMSSM. Specifically, we shall analyse decay processes involving gravitinos and calculate the corresponding life time. Depending on the strength of the conformal couplings, the width can be suppressed or enhanced significantly. In the case of enhancement the rapid decay of the gravitino implies a resolution of the gravitino overproduction, avoiding the Big-BangNucleosynthesis (BBN) constraints.

The structure of the article is as follows: in the next section III. we describe the basic Lagrangian formalism and properties underlying the conformal supergravity models of [1, 2]. In section [II we analyse the main decay processes involving gravitinos, calculate the associated widths (and lifetimes), and discuss how the latter are constrained by BBN. Conclusions and outlook are presented in section IV.

\section{LAGRANGIAN FORMALISM OF CONFORMAL SUPERGRAVITY MODELS}

The action of the conformal supergravity models of [1, 2], that we shall employ in our analysis below, in the Jordan frame reads:

$$
\begin{aligned}
& e^{-1} \mathcal{L}=-\frac{1}{6} \Phi\left[R(e)-\bar{\psi}_{\mu} R^{\mu}\right]-\frac{1}{6}\left(\partial_{\mu} \Phi\right)\left(\bar{\psi} \cdot \gamma \psi^{\mu}\right)+ \\
& +\mathcal{L}_{0}+\mathcal{L}_{1 / 2}+\mathcal{L}_{1}-V+\mathcal{L}_{m}+\mathcal{L}_{\text {mix }}+\mathcal{L}_{d}+\mathcal{L}_{4 \mathrm{f}}
\end{aligned}
$$

where the curvature $R(e)$ uses the torsionless connection $\omega_{\mu}^{a b}(e)$, with $e_{a}^{\mu}$ the vielbeins, and $e$ the vielbein determinant, and the gravitino kinetic term is defined using

$$
R^{\mu} \equiv \gamma^{\mu \rho \sigma}\left(\partial_{\rho}+\frac{1}{4} \omega_{\rho}^{a b}(e) \gamma_{a b}-\frac{3}{2} \mathrm{i} \mathcal{A}_{\rho} \gamma_{5}\right) \psi_{\sigma}
$$

Here $\mathcal{A}_{\mu}$ is the part of the auxiliary vector field containing only bosons, namely:

$$
\mathcal{A}_{\mu}=\frac{1}{6} \mathrm{i}\left(\partial_{\mu} z^{\alpha} \partial_{\alpha} \mathcal{K}-\partial_{\mu} \bar{z}^{\bar{\alpha}} \partial_{\bar{\alpha}} \mathcal{K}\right)-\frac{1}{3} A_{\mu}{ }^{A} P_{A},
$$

where $A_{\mu}{ }^{A}$ is the Yang-Mills gauge field, $z^{\alpha}$ are (complex) scalar fields, $\mathcal{K}(z, \bar{z})$ is the Kähler potential and $P_{A}$ is a momentum map or Killing potential, which encodes the non-Abelian gauge transformations on the scalars and may also include Fayet-Iliopoulos terms.

The notation $\mathcal{L}_{0}, \mathcal{L}_{1 / 2}$ and $\mathcal{L}_{1}$ denote respectively the 
kinetic terms of spin $0, \frac{1}{2}, 1$ fields in (1) [1]:

$$
\begin{aligned}
& \mathcal{L}_{0}=-\frac{1}{4 \Phi}\left(\partial_{\mu} \Phi\right)\left(\partial^{\mu} \Phi\right)+\frac{1}{3} g_{\alpha \bar{\beta}} \Phi\left(\hat{\partial}_{\mu} z^{\alpha}\right)\left(\hat{\partial}^{\mu} \bar{z}^{\bar{\beta}}\right), \\
& \mathcal{L}_{1 / 2}=-\frac{1}{2} \tilde{g}_{\alpha \bar{\beta}} \bar{\chi}^{\bar{\beta}} \not D \chi^{\alpha}+ \\
& \frac{1}{2} \Phi \bar{\chi}^{\alpha} \gamma^{\mu} \chi^{\bar{\beta}} \hat{\partial}_{\mu} z^{\gamma}\left[-\frac{1}{3} g_{\gamma \bar{\beta}} L_{\alpha}+\frac{1}{4} L_{\alpha \gamma} L_{\bar{\beta}}-\frac{1}{4} L_{\alpha} L_{\gamma \bar{\beta}}\right]+\text { h.c. } \\
& \mathcal{L}_{1}=\left(\operatorname{Re} f_{A B}\right)\left[-\frac{1}{4} F_{\mu \nu}^{A} F^{\mu \nu B}-\frac{1}{2} \bar{\lambda}^{A} \not D \lambda^{B}\right] \\
& +\frac{1}{4} \mathrm{i}\left[\left(\operatorname{Im} f_{A B}\right) F_{\mu \nu}^{A} \tilde{F}^{\mu \nu B}+\left(\hat{\partial}_{\mu} \operatorname{Im} f_{A B}\right) \bar{\lambda}^{A} \gamma_{5} \gamma^{\mu} \lambda^{B}\right]
\end{aligned}
$$

where $f_{A B}(z)$ is a holomorphic kinetic gauge matrix, $L_{\alpha} \equiv \partial_{\alpha} \ln (-\Phi), L_{\bar{\alpha}} \equiv \overline{L_{\alpha}}, L_{\alpha \beta}=\partial_{\alpha} L_{\beta}-\Gamma_{\alpha \beta}^{\gamma} L_{\gamma}$ and $g_{\gamma \bar{\beta}}=-\frac{1}{3} \Phi g_{\alpha \bar{\beta}}+\frac{1}{4} \Phi L_{\alpha} L_{\bar{\beta}}$, with $g_{\alpha \bar{\beta}}=\partial_{\alpha} \partial_{\bar{\beta}} \mathcal{K}$ is the Kähler metric, with the notation $\partial_{\alpha} \equiv \frac{\partial}{\partial z^{\alpha}}, \partial_{\bar{\alpha}} \equiv \frac{\partial}{\partial \bar{z}^{\alpha}}$.

In the notation of [1] , the covariant derivatives of the gauginos $\lambda^{A}$ are defined as:

$D_{\mu} \lambda^{A} \equiv\left(\partial_{\mu}+\frac{1}{4} \omega_{\mu}^{a b}(e) \gamma_{a b}-\frac{3}{2} \mathrm{i} \mathcal{A}_{\mu} \gamma_{5}\right) \lambda^{A}-A_{\mu}^{C} \lambda^{B} f_{B C}{ }^{A}$.

with $f_{A B}{ }^{C}$ the structure constants of the Non-Abelian gauge group.

The fermion mass terms, $\mathcal{L}_{m}$, including gravitino bare mass terms (if any) and the mixed terms $\mathcal{L}_{\text {mix }}$ containing scalars and fermions, including factors of the frame function, are given explicitly in [1], and again will not be of interest to us in this work. We shall be explicitly interested in the penultimate of the terms on the right-hand side of Eq. (11), namely:

$$
\begin{aligned}
& \mathcal{L}_{d}=\frac{1}{8}\left(\operatorname{Re} f_{A B}\right) \bar{\psi}_{\mu} \gamma^{a b}\left(F_{a b}^{A}+\widehat{F}_{a b}^{A}\right) \gamma^{\mu} \lambda^{B} \\
& +\frac{1}{\sqrt{2}}\left\{\bar{\psi}_{\mu} \gamma^{\nu} \gamma^{\mu} \chi^{\alpha}\left[\left(-\frac{1}{3} \Phi\right) g_{\alpha \bar{\beta}} \hat{\partial}_{\nu} \bar{z}^{\bar{\beta}}+\frac{1}{4} L_{\alpha} \partial_{\nu} \Phi\right]\right. \\
& \left.-\frac{1}{4} f_{A B} \bar{\chi}^{\alpha} \gamma^{a b} \widehat{F}_{a b}^{-A} \lambda^{B}-\frac{1}{3} \Phi L_{\alpha} \bar{\chi}^{\alpha} \gamma^{\mu \nu} D_{\mu} \psi_{\nu}+\text { h.c. }\right\}
\end{aligned}
$$

where

$$
\widehat{F}_{a b}{ }^{A} \equiv e_{a}{ }^{\mu} e_{b}{ }^{\nu}\left(2 \partial_{[\mu} A_{\nu]}^{A}+g f_{B C}{ }^{A} A_{\mu}^{B} A_{\nu}^{C}+\bar{\psi}_{[\mu} \gamma_{\nu]} \lambda^{A}\right)
$$

The explicit expression for the 4 -fermion terms $\mathcal{L}_{4 \mathrm{f}}$, which also contain a significant dependence on the frame function $\Phi$ and its derivatives, will be presented below. Such four-fermion terms, in particular four-gravitino ones, have been argued in [11] to play an important rôle in some variants of the above class of conformal supergravity models, which can characterise certain low-energy limits of superstring theories, in which the frame function $\Phi$ may be identified with the dilaton-axion complex superfield, $\frac{\Phi}{3} \equiv \frac{1}{\kappa^{2}} e^{-2 \varphi}$. Such models can serve as prototypes in which the Deser-Zumino [12] mechanism for $d y$ namical breaking of local supersymmetry (supergravity) scenario is realised explicitly. The important point to notice in these class of theories is the presence of the frame function $\Phi$ in front of the four-gravitino terms. These imply that, depending on the value of $\Phi$, assumed to be stabilised appropriately by rolling to the minimum of an appropriate dilaton potential (generated by e.g. by string loops, in case one embeds such conformal SUGRA models to string theory, or other ways of breaking the scale symmetry), the effective coupling of the four-gravitino interactions can be much larger than the gravitational coupling. Indeed, in the Einstein frame (denoted by a suffix E), the graviton field (in the original Jordan frame, denoted by a suffix $J$ ) is redefined by means of

$$
g_{J}^{\mu \nu}=e^{-2 \varphi} g_{E}^{\mu \nu}=\left(\frac{\kappa^{2}}{3} \Phi\right)_{\text {bosonic }} g_{E}^{\mu \nu}, \quad\left(\left.\Phi\right|_{\text {bosonic }}>0\right),
$$

so that the curvature term in the target-space supergravity action has the canonical form, with coefficient the gravitational coupling $\kappa^{2}=8 \pi G_{N}$. with $G_{N}$ Newton's (four-dimensional) gravitational constant.

In the Einstein frame, where we shall work for the purposes of this work, the gravitational part of the effective conformal supergravity action, including the fermionic torsion induced four-gravitino terms reads

$$
\begin{aligned}
& \mathcal{L}^{E}\left(e^{E}\right)^{-1}=-\frac{1}{2 \kappa^{2}} R^{E}\left(e^{E}\right)+\frac{1}{2} \epsilon^{\mu \nu \rho \sigma}{\overline{\psi^{\prime}}}_{\mu} \gamma_{5} \gamma_{\nu} D_{\rho}^{E} \psi_{\sigma}^{\prime}{ }_{\sigma} \\
& e^{2 \varphi} V^{E}+\frac{11 \kappa^{2}}{16} e^{-2 \varphi}\left[\left(\overline{\psi^{\prime}}{ }_{\mu} \psi^{\prime \mu}\right)^{2}-\left(\overline{\psi^{\prime}}{ }_{\mu} \gamma_{5} \psi^{\prime \mu}\right)^{2}\right]+ \\
& \frac{33}{64} \kappa^{2} e^{-2 \varphi}\left(\overline{\psi^{\prime}}{ }^{\rho} \gamma_{5} \gamma_{\mu} \psi^{\prime}{ }_{\rho}\right)^{2}+\cdots= \\
& -\frac{1}{2 \kappa^{2}} R^{E}\left(e^{E}\right)+\frac{1}{2} \epsilon^{\mu \nu \rho \sigma} \overline{\psi^{\prime}}{ }_{\mu} \gamma_{5} \gamma_{\nu} D_{\rho}^{E} \psi_{\sigma}^{\prime}{ }_{\sigma} \\
& e^{2 \varphi} V^{E}+\rho^{2}(x)+\frac{\sqrt{11}}{2} \kappa \rho(x) e^{-\varphi}\left(\overline{\psi^{\prime}}{ }_{\mu} \psi^{\prime \mu}\right)+ \\
& \pi^{2}(x)+\frac{\sqrt{11}}{2} e^{-\varphi} \kappa i \pi(x)\left(\overline{\psi^{\prime}}{ }_{\mu} \gamma_{5} \psi^{\prime \mu}\right)+ \\
& \frac{\sqrt{33}}{2} \kappa e^{-\varphi} i \lambda^{\nu}\left({\overline{\psi^{\prime}}}^{\rho} \gamma_{5} \gamma_{\nu} \psi_{\rho}^{\prime}{ }_{\rho}\right)+\ldots,
\end{aligned}
$$

where $R(e)$ denotes the cirvature term with respect to the torsion-free spin connection, $\psi_{\mu}^{\prime}$ denotes the canonicallynormalised gravitino with standard kinetic term as in $N=1$ supergravity,

$$
\psi_{\mu}^{\prime}=e^{\varphi} \psi_{\mu}
$$

while the ... denote structures, including auxiliary fields, that are not of direct interest to us here. In writing (9) we have expanded the four-gravitino terms into detailed structures to exhibit explicitly the terms that generate masses, and we linearise the four-gravitino terms. The condensate of interest to us is the v.e.v. of the linearizing field $\rho(x)$.

The reader should notice that the coefficients of the gravitino- $\rho$ interaction terms in (9) contain dilatondependent factors $\sim e^{-\varphi}$, and are thus proportional, not to $\kappa$, but to:

$$
\tilde{\kappa} \equiv \kappa e^{-\varphi}
$$


A one-loop analysis shows that the effective potential for the condensate $\rho$ field acquires a minimum at [11]

$$
\rho_{\min }=\langle\rho\rangle= \pm 0.726
$$

at which it vanishes. The gravitino mass term, then, in (9) then takes the following form:

$$
-m_{3 / 2} \bar{\psi}_{\mu}^{\prime} \Gamma^{\mu \nu} \psi_{\nu}^{\prime}=-\frac{1}{2} m_{3 / 2} \bar{\psi}_{\mu}^{\prime} \psi^{\prime \mu},
$$

with the dynamically-generated gravitino mass of order

$$
m_{3 / 2}=\sqrt{11} \tilde{\kappa}^{-1} \rho_{\min }=2.408 \kappa^{-1} e^{\varphi}=\frac{2.408}{\sqrt{8 \pi}} e^{\varphi} M_{P} .
$$

For large negative values of the v.e.v. $\langle\varphi\rangle<0$ the resulting gravitino mass is mauch smaller than the Planck scale, and thus the effective coupling $\tilde{\kappa}$ (11) is much larger than the gravitational coupling $G_{N}$.

This implies that quantum gravitational corrections to the Minkowski space-time background, on which the above minimisation of the effective potential has been considered are not strong enough to destabilise (at least quickly) the gravitino condensate, unlike the case of standard $\mathrm{N}=1$ supergravity [13]. This prompted the authors of 11] to consider large positive values of

$$
\left\langle e^{-2 \varphi}\right\rangle \equiv \frac{1}{3} \kappa^{2}\left\langle\left.\Phi\right|_{\text {bosonic }}\right\rangle \gg 1,
$$

and discuss their relevance to the above-mentioned scenario of dynamical metastable breaking of local Sipersymmetry (SUSY) and generation of gravitino mass.

It is the point of this article to examine the constraints implied by such an assumption on the dark sector of the Universe in the case of the NMSSM embedded in this conformal supergravity framework. However, we shall not only restrict ourselves to the case of negative expectation values of the dilaton, but we shall be more general and also consider the dark-matter phenomenology/cosmology of the case of positive dilaton v.e.v. In this latter case, dynamical breaking of local SUSY is not possible in view of the destabilising effects of the graviton fluctuations, nevertheless one assumes conventional breaking of SUSY, e.g. through gluino condensation [14], which is then communicating to the gravity sector to result in a non-trivial gravitino mass term $m_{3 / 2}$.

\section{DECAY PROCESSES INVOLVING GRAVITINOS AND ASSOCIATED CONSTRAINTS}

We first notice that, in the Einstein frame, one has to first normalise the kinetic terms of the scalars $z^{\alpha}$ and $\chi$ and $\lambda$ fermions, by appropriate redefinitions involving the frame function $\Phi$. In particular the gauginos should be renormalised in the Einstein frame as

$$
\lambda^{\prime}=e^{2 \varphi} \lambda
$$

in order to acquire canonical kinetic terms. On the other hand the gauge terms of the spin- 1 part $\mathcal{L}_{1}$ (4) are already in canonical form, in view of the conformal nature of the (Maxwell-like) kinetic terms for the Yang Mills fields. Here we consider the case $f_{A B}=$ const $=1$.

Taking (10) and (16) into account, as well as the fact that the Yang-Mills gauge fields are not renormalised in the Einstein frame by the frame function $e^{-2 \varphi}$, we may write the first term of the right-hand side of (6) in the Einstein frame as:

$$
\left(L_{d}\right)_{E}=\frac{1}{8}\left(\operatorname{Re} f_{A B}\right) \bar{\psi}_{\mu}^{\prime} \gamma^{a b}\left({F^{\prime}}_{a b}^{A}+{\widehat{F^{\prime}}}_{a b}^{A}\right) \gamma^{\mu} \lambda^{\prime B} .
$$

This term is responsible for the gravitino-gaugino-gaugeboson interaction and it does not transformed going to the new frame.

Tying to recap the transformations we have

$$
\begin{aligned}
\psi_{\mu}^{\prime} & =e^{\varphi} \psi_{\mu} \\
\lambda^{\prime} & =e^{2 \varphi} \lambda .
\end{aligned}
$$

The scalar and vector fields don't change. Using these we can calculate how the interactions that are relevant to the gravitino decays change due to the dilation presence. In particular we are interested in the $\psi \chi Z(\gamma)$ decays, where with $\chi$ we denote the neutralino that is the LSP in our model. Doing so, we may then consider the terms $\mathcal{L}_{d}$ (66) in order to compute the decay rate of the massive gravitino field $\tilde{G}$ into, say, a neutralino $\chi$ in the NMSSM and a $Z$ gauge boson:

$$
\tilde{G} \rightarrow \chi+Z .
$$

The so affected gravitino decay rate, will in turn affect the Dark Matter relic density (assumed to be dominated by neutralinos in NMSSM) and this may imply stronger Big Bang Nucleosynthesis (BBN) constraints. It is therefore important that detailed cosmological studies of such dilaton extended mSUGRA models are performed.

In the usual mSUGRA gravitino satisfies the RaritaSchwinger (RS) eqs.

$$
\begin{aligned}
\gamma^{\mu} \psi_{\mu}(x) & =0 \\
(i \not \partial-m) \psi_{\mu}(x) & =0
\end{aligned}
$$

which result from the RS action 15]

$$
\mathcal{L}=-\frac{1}{2} \epsilon^{\mu \nu \rho \sigma} \bar{\psi}_{\mu} \gamma_{5} \gamma_{\nu} \partial_{\rho} \psi_{\sigma}-\frac{1}{4} m_{3 / 2} \bar{\psi}_{\mu}\left[\gamma^{\mu}, \gamma^{\nu}\right] \psi_{\nu} .
$$

The RS action including the dilaton effects can be written as

$$
\mathcal{L}^{\prime}=-\frac{1}{2} \epsilon^{\mu \nu \rho \sigma} \bar{\psi}_{\mu}^{\prime} \gamma_{5} \gamma_{\nu} \partial_{\rho} \psi_{\sigma}^{\prime}-\frac{1}{4} m_{3 / 2}^{\prime} \bar{\psi}_{\mu}^{\prime}\left[\gamma^{\mu}, \gamma^{\nu}\right] \psi_{\nu}^{\prime}
$$

where $\psi_{\mu}^{\prime} \rightarrow e^{\varphi} \psi_{\mu}$, and the relation between $m_{3 / 2}^{\prime}$ to $m_{3 / 2}$ is

$$
m_{3 / 2}^{\prime}=e^{\varphi} m_{3 / 2}
$$


so for $\langle\varphi\rangle<0$, which is the physical case in several of the backgrounds discussed in order to allow for dynamical breaking of local SUSY, the gravitino mass in the conformal supergravity scenario will be smaller than the corresponding one in the normal SUGRA.

In the NMSSM [10] the neutralino field can be written as:

$$
\chi=N_{11} \tilde{B}+N_{12} \tilde{W}_{0}^{3}+N_{13} \tilde{H}_{0}^{1}+N_{14} \tilde{H}_{0}^{2}+N_{15} \tilde{S},
$$

where $N_{i j}$ are the elements of the $5 \times 5$ neutralino diagonalizing matrix. If $N_{11}$ dominates the sum $\sum_{i=1,5} N_{i 1}^{2}=$ 1 , then the lightest neutralino is bino-like. On the other hand, if $N_{15}$ is dominant then the neutrslino is singlinolike. The latest data of the LHC experiments, indicating a Higgs boson mass in the ballpark of $125 \mathrm{GeV}$ [16], combined with other experimental data from B-physics and direct dark matter searches, seem to disfavor the singlino case [17]. Thus, in the following it will be assumed that the lightest neutralino is mainly bino. In this case, the dominant two body decay channels for gravitino are $\tilde{G} \rightarrow \gamma \chi$ and $\tilde{G} \rightarrow Z \chi$. Nevertheless, even in the singlino-like case those channels, especially the $\gamma \chi$, are dominant, mainly due to the large available phase space. Therefore, our assumption that the light neutralino is mainly bino is sufficiently generic.

We note in passing at this point that, as shown in [18], a time-dependent (cosmological) dilaton (which can run with the cosmic time before BBN) can reduce considerably the neutralino relic density, thereby increasing the cosmologically-allowed available parameter space of SUSY even beyond the LHC reach. In this article we ignore such effects, focusing on the gravitino interactions exclusively, and assuming that the dilaton in our case has been stabilised to its vacuum expectation value at the scale of SUSY breaking (or at least it is approximately constant during a cosmological epoch). However, even for stabilised dilatons, in the Einstein frame, the neutralino-pair annihilation processes are affected by the conformal couplings, in particular are enhanced for large $e^{\varphi}>1$. Such enhancement may reduce the relic abundances already in the constant dilaton case. We postpone a comprehensive study of such effects on gravitino decays and the neutralino dark matter abundance for a future study.

Below we shall consider two cases: one, in which the neutralino is the stable Dark Matter candidate and the gravitino is heavier, thus unstable, and the other, in which the gravitino is cosmologically stable and thus constitutes the dark matter candidate. We commence our discussion from the former case.

In such a case, the formula for the decay width $\tilde{G} \rightarrow \gamma \chi$ without the dilaton effects, reads as [19]:

$$
\Gamma_{\gamma \chi}=\frac{1}{16 \pi} \frac{\overline{\left|\mathcal{M}_{\gamma}\right|^{2}}}{m_{3 / 2}} \mathcal{F}\left(m_{3 / 2}, m_{\chi}, 0\right),
$$

where the spin average amplitude squared is

$$
\overline{\left|\mathcal{M}_{\gamma}\right|^{2}}=\frac{B_{\gamma}^{2}}{6 M_{P}^{2}} \frac{1}{m_{3 / 2}^{2}}\left(m_{3 / 2}^{2}-m_{\chi}^{2}\right)^{2}\left(3 m_{3 / 2}^{2}+m_{\chi}^{2}\right)
$$

and the kinematical factor is defined as

$$
\begin{aligned}
\mathcal{F}\left(m_{0}, m_{1}, m_{2}\right) & =\frac{1}{m_{0}^{2}}\left[\left(m_{0}^{2}-\left(m_{1}+m_{2}\right)^{2}\right)\right. \\
& \left.\times\left(m_{0}^{2}-\left(m_{1}-m_{2}\right)^{2}\right)\right]^{1 / 2} .
\end{aligned}
$$

On the other hand, taking into account the dilaton effects, i.e. considering the corresponding process in the conformal SUGRA model in the Einstein frame, the corresponding width becomes

$$
\Gamma_{\gamma \chi}^{\prime}=\frac{1}{16 \pi} \frac{\overline{\left|\mathcal{M}_{\gamma}^{\prime}\right|^{2}}}{c m_{3 / 2}} \mathcal{F}\left(c m_{3 / 2}, m_{\chi}, 0\right),
$$

where

$$
\begin{aligned}
& \overline{\left|\mathcal{M}_{\gamma}^{\prime}\right|^{2}}=\frac{B_{\gamma}^{2}}{12 M_{P}^{2}} \frac{1}{m_{3 / 2}^{2}} c^{2}\left(m_{3 / 2}^{2}-m_{\chi}^{2}\right)^{2} \\
& \times\left(6 c^{4} m_{3 / 2}^{2}+\left(c^{2}+1\right) m_{\chi}^{2}\right) .
\end{aligned}
$$

Above it was defined $c=e^{\varphi}$. Notice that putting $c=1$ we recover the result of Eq. (24). The factor $B_{\gamma}$ is related to the bino $(\tilde{B})$ and neutral wino $\left(\tilde{W}_{0}^{3}\right)$ components of the neutralino, that is $B_{\gamma}=N_{i 1} \cos \theta_{W}+N_{i 2} \sin \theta_{W}$, where $\theta_{W}$ is the electroweak mixing angle.

For the channel $\tilde{G} \rightarrow Z \chi$ in the standard (dilaton-free) SUGRA the width reads as:

$$
\Gamma_{Z \chi}=\frac{1}{16 \pi} \frac{\overline{\left|\mathcal{M}_{Z}\right|^{2}}}{m_{3 / 2}} \mathcal{F}\left(m_{3 / 2}, m_{\chi}, M_{Z}\right),
$$

where

$$
\begin{aligned}
\overline{\left|\mathcal{M}_{Z}\right|^{2}} & =\frac{B_{Z}^{2}}{6 M_{P}^{2}} \frac{1}{m_{3 / 2}^{2}}\left[3 m_{3 / 2}^{6}-m_{3 / 2}^{4}\left(5 m_{\chi}^{2}+M_{Z}^{2}\right)\right. \\
& +12 m_{3 / 2}^{3} m_{\chi} M_{Z}^{2}+m_{3 / 2}^{2}\left(m_{\chi}^{4}-M_{Z}^{4}\right) \\
& \left.+\left(m_{\chi}^{2}-M_{Z}^{2}\right)^{3}\right]
\end{aligned}
$$

where $B_{Z}=-N_{i 1} \sin \theta_{W}+N_{i 2} \cos \theta_{W}$. In the case of dilaton the same width becomes

$$
\Gamma_{Z \chi}^{\prime}=\frac{1}{16 \pi} \frac{\overline{\left|\mathcal{M}_{Z}^{\prime}\right|^{2}}}{c m_{3 / 2}} \mathcal{F}\left(c m_{3 / 2}, m_{\chi}, M_{Z}\right)
$$


where

$$
\begin{aligned}
& \overline{\left|\mathcal{M}^{\prime}\right|^{2}}=\frac{B_{Z}^{2}}{24 M_{P}^{2}} \frac{1}{m_{3 / 2}^{2}} \\
\times & c^{2}\left[-2\left(c^{2}+1\right) M_{Z}^{6}+M_{Z}^{4}\left(6\left(c^{2}+1\right) m_{\chi}^{2}\right.\right. \\
+ & \left.\left(-6 c^{4}+c^{2}+1\right) m_{3 / 2}^{2}\right) \\
+ & 2\left(m_{3 / 2}-m_{\chi}\right)^{2}\left(m_{3 / 2}+m_{\chi}\right)^{2}\left(6 c^{4} m_{3 / 2}^{2}+\left(c^{2}+1\right) m_{\chi}^{2}\right) \\
+ & M_{Z}^{2}\left(-6\left(c^{2}+1\right) m_{\chi}^{4}\right. \\
+ & \left(-6 c^{4}+c^{2}+1\right) m_{3 / 2}^{4}+6 c\left(12 c^{4}-3 c^{2}-1\right) m_{3 / 2}^{3} m_{\chi} \\
+ & \left.\left.3\left(-2 c^{4}+c^{2}+1\right) m_{3 / 2}^{2} m_{\chi}^{2}\right)\right] .
\end{aligned}
$$

It is worth noticing that $\Gamma_{Z \chi}$ goes to $\Gamma_{\gamma \chi}$ in the limit $M_{Z} \rightarrow 0$, and the same holds for $\Gamma^{\prime}$.

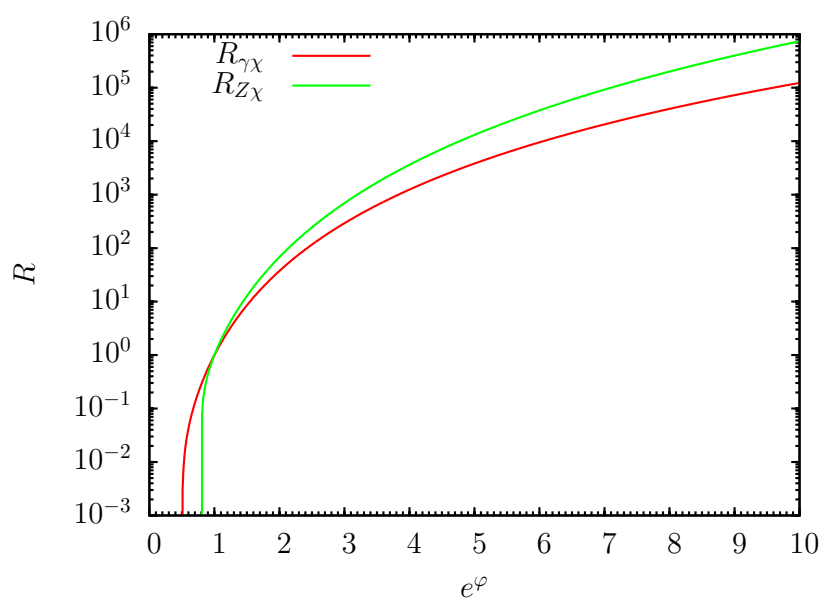

FIG. 1: The ratio $R=\Gamma^{\prime} / \Gamma$ for the channels $\tilde{G} \rightarrow \chi \gamma$ and $\tilde{G} \rightarrow \chi Z$, as a function of $e^{\varphi}$, for the neutralino dark matter case.

We start our numerical analysis discussing models where dark matter consists of neutralinos and the gravitino is unstable. In this case decays of the gravitinos to neutralinos can be an important constraint, affecting significantly the BBN predictions. In Fig. 1 we present the ratio $R=\Gamma^{\prime} / \Gamma$ for the processes $\tilde{G} \rightarrow \gamma \chi$ and $\tilde{G} \rightarrow Z \chi$ as a function of $e^{\varphi}$. To make this figure we use the numerical values $m_{3 / 2}=300 \mathrm{GeV}, m_{\chi}=150 \mathrm{GeV}$, and for these the two body decay widths for the dominant channels (involving $\gamma$ ) are $1.4 \times 10^{-32} \mathrm{GeV}$ and $3.3 \times 10^{-33}$ $\mathrm{GeV}$ for the $\gamma \chi$ and $Z \chi$, respectively. This yields a gravitino lifetime $\sim 4.7 \times 10^{7} \mathrm{~s}$.

We first concentrate in the region of the Fig. 1 where $c>1$, for which although dynamical generation of gravitino mass and thus breaking of local SUSY may not occur [11, 13] nevertheless, as mentioned above, one may assume a more or less conventional mechanism [14] for SUSY breaking and the generation of a gravitino mass term $m_{3 / 2}$, (21), (22). In this case, there is an enhancement of the ratio $R=\Gamma^{\prime} / \Gamma$, where the prime denotes the width for the conformal SUGRA case, where dilaton effects are taken into account. An important observation concerns the fact that for $c<1$, which is the case where dynamical (metastable) breaking of local SUSY mass be possible, according to the arguments of [11] reviewed above ( $c f$. discussion leading to Eq. (14)) the decay of gravitino to photons and neutralinos is kinematically forbidden. This case will face important constraints from BBN which will be discussed below.

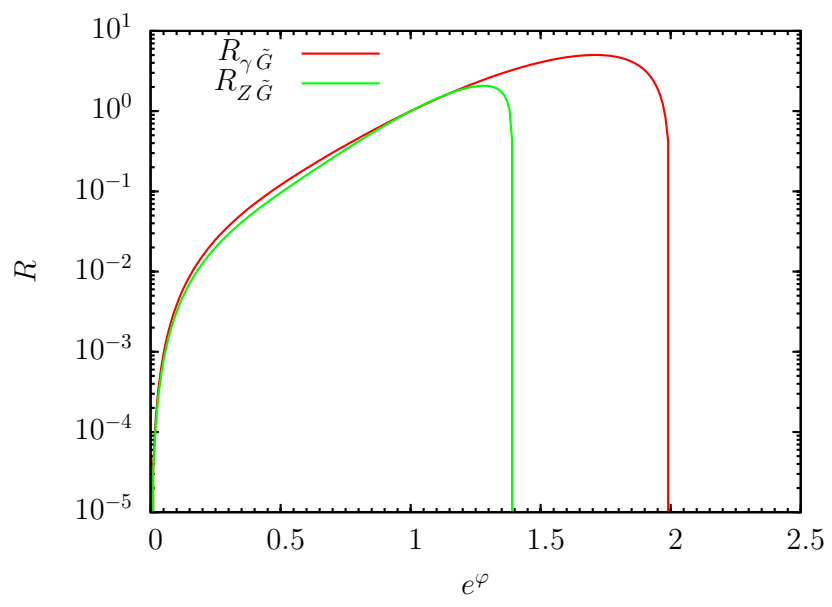

FIG. 2: The ratio $R=\Gamma^{\prime} / \Gamma$ for the channels $\chi \rightarrow \tilde{G} \gamma$ and $\chi \rightarrow \tilde{G} Z$, as a function of $e^{\varphi}$ for the gravitino dark matter case.

Concerning the BBN constraints, we know that they become important for lifetime of the unstable particle $\tau \gtrsim 10^{2} \mathrm{~s}[20]$. Since $\tau^{\prime}=\tau / R$ and the gravitino lifetime without the dilaton effects is $\mathcal{O}\left(10^{7}\right) \mathrm{s}$, one observes that with $R \gtrsim 10^{5}$ one avoids all the important BBN constraints practically for any gravitino mass. Using Fig. 1 we understand that this happens for $e^{\varphi} \gtrsim 9$ or 10 . For smaller values of $e^{\varphi}$ the BBN appears to become important, since the lifetime of the gravitino approaches its original value $10^{7} \mathrm{~s}$ as $e^{\varphi} \rightarrow 1$, but its abundance is still enhanced by the factor $e^{\varphi}$. On the other hand, values for $e^{\varphi}<1$ enhance dramatically the gravitino lifetime, leading to the exclusion of this range of the scale factor, being incompatible with the BBN constraints. We have checked numerically different values of gravitino mass up to $\mathrm{TeV}$, but our results remain basically the same. That is, one finds that for $e^{\varphi}$ in the range above 8 or 10 , the BBN constraints can be avoided.

In addition, we discuss also the complementary case where the gravitino is the LSP. In this case one studies the reverse processes $\chi \rightarrow \tilde{G} \gamma$ and $\chi \rightarrow \tilde{G} Z$, from the point of view of the constraints induced by BBN. To compute the corresponding decay widths we use the fact that the amplitudes squared $\overline{\left|\mathcal{M}_{\gamma, Z}\right|^{2}}$, both for the standard and the dilaton cases, are the same as before, due to the assumed CPT invariance and unitarity. On the other hand, one has to interchange $m_{\chi}$ and $m_{3 / 2}$ in 
$\mathcal{F}$ and in the denominators of Eqs. (24), (27), (29), and (31). Doing so, we plot in Fig. 2 the ratio $R=\Gamma^{\prime} / \Gamma$ for these reverse processes. The numerical values we use are $m_{3 / 2}=150 \mathrm{GeV}, m_{\chi}=300 \mathrm{GeV}$, exactly the reverse case of Fig. 1. With this choice, the neutralino lifetime is now $\mathcal{O}\left(10^{7}\right) \mathrm{s}$. We thus see that the kinematically allowed region happens for values of $c=e^{\varphi}<2$. This region depends on the choice neutralino and gravitino masses, but the attained values of $R$ are not sensitive to this. One observes that in this case $R \lesssim 10$. Thus in this case the dilaton effects can not be used to relax the BBN constraints.

\section{CONCLUSIONS AND OUTLOOK}

In this article we have discussed the effects of stabilised dilatons on processes involving unstable particles, including gravitinos, that may affect BBN in conformal SUGRA models, incorporating the NMSSM in their spectra. We have found that, in the case where the gravitino is unstable, and the neutralino plays the role of dark matter, there are regions of the scale (dilaton) factor $e^{\varphi}>8$ in which the BBN constraints can be avoided altogether. Moreover, in such regimes the neutralino dark matter abundances may be diluted thereby avoiding the cosmological and particle physics constraints on SUSY matter at current colliders, including LHC. Unfortunately, this case seems not to favour dynamical SUGRA breaking (due to quantum-gravity instabilities), and therefore one has to assume more-or-less conventional breaking of SUSY and its communication to the gravitational sector. On the other hand, if the gravitino is cosmologically stable, playing the role of dark matter, the BBN constraints are very restrictive in the full (kinematicallyallowed) range of the scale (dilaton) factor $e^{\varphi}$.

We have not discussed in detail time-dependent dilaton effects that involve running scale factors up to BBN, which are known to reduce significantly the dark matter abundances. Such effects, when combined with the dilaton effects on the gravitino decays considered here, may change significantly the cosmology and phenomenology of such conformal SUGRA models. We plan to return to these interesting issues in a forthcoming publication.

\section{Acknowledgements}

The work of N.E.M. is supported in part by STFC (UK) under the research grant ST/J002798/1 and by the London Centre for Terauniverse Studies (LCTS), using funding from the European Research Council via the Advanced Investigator Grant 267352; this latter grant also supported visits by V.C.S. to the CERN TH Division, which he thanks for its hospitality and where this work was initiated. The work of V.C.S. is supported by Marie Curie International Reintegration grant SUSYDMPHEN, MIRG-CT-2007-203189.
[1] S. Ferrara, R. Kallosh, A. Linde, A. Marrani and A. Van Proeyen, Phys. Rev. D 82, 045003 (2010) arXiv:1004.0712 [hep-th]].

[2] S. Ferrara, R. Kallosh, A. Linde, A. Marrani and A. Van Proeyen, Phys. Rev. D 83, 025008 (2011) arXiv:1008.2942 [hep-th]].

[3] F. L. Bezrukov and M. Shaposhnikov, Phys. Lett. B 659, 703 (2008) arXiv:0710.3755 [hep-th]].

[4] F. Bezrukov and M. Shaposhnikov, JHEP 0907, 089 (2009) arXiv:0904.1537 [hep-ph]].

[5] F. Bezrukov, A. Magnin, M. Shaposhnikov and S. Sibiryakov, JHEP 1101, 016 (2011) arXiv:1008.5157 [hep-ph]].

[6] J. Garcia-Bellido, J. Rubio, M. Shaposhnikov and D. Zenhausern, arXiv:1107.2163 [hep-ph].

[7] M. B. Einhorn and D. R. T. Jones, JHEP 1003, 026 (2010) arXiv:0912.2718 [hep-ph]].

[8] L. Alvarez-Gaume, C. Gomez and R. Jimenez, Phys. Lett. B 690, 68 (2010) arXiv:1001.0010 [hep-th]].

[9] L. Alvarez-Gaume, C. Gomez and R. Jimenez, JCAP 1103, 027 (2011) arXiv:1101.4948 [hep-th]].

[10] C. Panagiotakopoulos and K. Tamvakis, Phys. Lett. B 446, 224 (1999) hep-ph/9809475; M. Maniatis, Int. J. Mod. Phys. A 25, 3505 (2010) arXiv:0906.0777 [hep-ph]]; For a review see: U. Ellwanger, C. Hugonie and A. M. Teixeira, Phys. Rept. 496, 1 (2010)
arXiv:0910.1785 [hep-ph]] and references therein.

[11] J. Ellis and N. E. Mavromatos, Phys. Rev. D 84, 085016 (2011) arXiv:1108.0877 [hep-th]].

[12] S. Deser and B. Zumino, Phys. Rev. Lett. 38, 1433 (1977).

[13] I. L. Buchbinder and S. D. Odintsov, Class. Quant. Grav. 6, 1955 (1989).

[14] see, for instance: H. P. Nilles, Phys. Rept. 110, 1 (1984) and references therein.

[15] W. Rarita and J. Schwinger, Phys. Rev. 60, 61 (1941).

[16] G. Aad et al. [ATLAS Collaboration], Phys. Lett. B 716, 1 (2012) arXiv:1207.7214 [hep-ex]]; S. Chatrchyan et al. [CMS Collaboration], Phys. Lett. B 716, 30 (2012) arXiv:1207.7235 [hep-ex]].

[17] K. Kowalska, S. Munir, L. Roszkowski, E. M. Sessolo, S. Trojanowski and Y. -L. S. Tsai, arXiv:1211.1693 [hep$\mathrm{ph}]$.

[18] A. B. Lahanas, N. E. Mavromatos and D. V. Nanopoulos, PMC Phys. A 1, 2 (2007) hep-ph/0608153; Phys. Lett. B 649, 83 (2007) hep-ph/0612152; A. B. Lahanas, Phys. Rev. D 83, 103523 (2011) arXiv:1102.4277 [hepph]]; A. B. Lahanas and V. C. Spanos, JHEP 1206, 089 (2012) arXiv:1201.2601 [hep-ph]].

[19] J. R. Ellis, K. A. Olive, Y. Santoso and V. C. Spanos, Phys. Lett. B 588, 7 (2004) hep-ph/0312262.

[20] M. Kawasaki, K. Kohri and T. Moroi, Phys. Rev. D 71, 
083502 (2005) astro-ph/0408426; R. H. Cyburt, J. Ellis, B. D. Fields, F. Luo, K. A. Olive and V. C. Spanos, JCAP
0910, 021 (2009) arXiv:0907.5003 [astro-ph.CO]]. 